\title{
Evidence for a Broad Autism Phenotype
}

\author{
Kristel De $\operatorname{Groot}^{1}$ (D) - Jan W. Van Strien ${ }^{1}$
}

Published online: 24 May 2017

(C) The Author(s) 2017. This article is an open access publication

\begin{abstract}
The broad autism phenotype implies the existence of a continuum ranging from individuals displaying almost no autistic traits to severely impaired diagnosed individuals. Recent studies have linked this variation in autistic traits to several domains of functioning. However, studies focusing on social-communicational traits associated with autism often suffer from two problems. First, they examine very specific behaviours, not taking the broad range of behaviours social functioning is comprised of into account. Second, most studies compare individuals scoring at the upper and lower extremes of the continuum, neglecting the natural range of autistic trait scores. The present study accommodates for these limitations by examining the link between self-reported autistic traits and a broad self-report measure of social functioning across individuals exhibiting a natural range of autistic traits. The results show that after tackling the discussed limitations, autistic traits still predict the amount of social behaviour people exhibit and the level of discomfort they experience when doing so. The amount of social behaviour and the experienced discomfort were especially related to autistic traits in the social and attention switching domains. The findings were still significant after controlling for the conceptual overlap with the social domain of the autism measure. These findings support the broad autism phenotype by showing how a continuous measure of autistic traits is related to a continuous measure of social functioning.
\end{abstract}

Kristel De Groot

k.degroot@ese.eur.nl

1 Faculty of Social Sciences, Erasmus University Rotterdam, Burgemeester Oudlaan 50, 3062 PA Rotterdam, The Netherlands
Keywords Autism spectrum disorder (ASD) - Broad autism phenotype $\cdot$ Social functioning $\cdot$ Autism-spectrum quotient (AQ) $\cdot$ Autism spectrum hypothesis

\section{Introduction}

Although mental disorders are mostly diagnosed in a categorical manner, practitioners and researchers have long recognised the possibility of diagnosing across a dimension 'in which the imperceptible change from the realm of normal mental life to that of obvious derangement takes place' (Kraepelin 1917, p. 295). Although the dimensional view has not won ground for most disorders, the change from the fourth to the fifth edition of the Diagnostic and Statistical Manual of mental disorders (DSM) did allow for one disorder to be recognised as existing on a spectrum: autism. While the DSM-IV-TR (American Psychiatric Association 2000) used the term 'autistic disorder' and specified five separate sub-groups, the DSM-5 (American Psychiatric Association 2013) acknowledged that individuals with autism fall on a spectrum according to their level of functional independence, and changed the diagnostic name accordingly to 'autism spectrum disorder' (ASD). Within this concept, individuals show varying degrees of severity on the main symptoms: difficulties in social communication, restricted and repetitive behaviour, preference for sameness and routines and sensory abnormalities.

A strong extension of the dimensional approach for autism concerns the autism spectrum hypothesis, which proposes a broadening of the spectrum so that not only autistic traits in the clinical population but also within the general population are included (Bailey et al. 1998; Baron-Cohen et al. 2001; Hoekstra et al. 2008; Wing 1988). This extension of the autistic trait continuum into the general population implies the existence of a broad autism phenotype: a continuum that ranges from individuals who display almost no autistic traits 
to diagnosed individuals who are severely impaired. Every single individual exists somewhere on that spectrum, and the amount of autistic traits one exhibits is hypothesised to be correlated with several domains of functioning, such as cognition, motor skill and social functioning.

The concept of a broad autism phenotype was first suggested by studies indicating that relatives of individuals diagnosed with ASD are more likely to express mild autistic traits, regardless of diagnosis (see Bailey et al. 1998, for a review). In addition, autism occurs more often in families of individuals studying or working in fields that depend less on 'folk psychology' (understanding of people's behaviour and intentions) but that do require advanced 'folk physics' (understanding of objects in terms of their mechanical properties), such as engineering, physics and mathematics (Baron-Cohen et al. 1997; Baron-Cohen et al. 1998). Further support for the existence of a broad autism phenotype comes from studies focusing on genetics. An epidemiological study by Constantino and Todd (2003) showed that relatives of probands with ASD have higher chances of exhibiting autistic symptoms below diagnostic threshold, thereby supporting the proposition that autism is not a discrete disorder. This was confirmed by gene studies indicating that the genetic variants that put an individual at risk for autism are expressed as milder, qualitatively similar characteristics that together form the broad autism phenotype (Piven 2001; Szatmari et al. 2007). What is inherited is not necessarily clinically diagnosable ASD, but a broad phenotype that is characterised by a symptom continuity ranging from typicality to autism. This explains why the disorder itself is not often observed in family members, whereas sub-clinical characteristics are (Bishop et al. 2004; Ruta et al. 2012; Ruzich et al. 2016; Wheelwright et al. 2010).

Building upon the autism spectrum hypothesis, Baron-Cohen et al. (2001) developed a brief, self-administered instrument for measuring the degree to which an adult of normal intelligence possesses autistic traits: the Autism-Spectrum Quotient (AQ). The AQ has given impetus to a large amount of research examining the broad autism phenotype. Many studies show that a higher degree of ASD traits in typically developing (TD) individuals predicts symptoms similar to but milder than those seen in diagnosed patients, thereby supporting the view that autistic traits are continuously distributed throughout the general population. Most of this research has focused on the cognitivebehavioural characteristics found in ASD. Similar to diagnosed individuals (Shah and Frith 1983, 1993), TD individuals with high-AQ scores are faster and more accurate on tasks measuring visuospatial ability, such as the Embedded Figures Test and the Block Design Task (Grinter et al. 2009; Stewart et al. 2009; but see Scheeren and Stauder 2008). In addition, they perform well on especially the visuospatial items of Raven's Advanced Progressive Matrices, indicating enhanced perceptual functioning (Fugard et al. 2011). Also, Figure-Ground visual skill scores are positively correlated with (not AQ-measured) self-reported autism-like social features (DiCriscio and Troiani 2017). Metaanalytic results also showed superior visual search within the broad autism phenotype (Cribb et al. 2016). Furthermore, TD individuals with more self-reported autistic traits showed a Flanker task interference pattern similar to that of diagnosed individuals, having stronger interference at higher perceptual loads (Bayliss and Kritikos 2011; Remington et al. 2009). Finally, the bias many individuals with ASD show towards repetitive behaviour and the motor problems that are often observed in ASD also carry over into the general population (Poljac et al. 2012; Ridley et al. 2011). However, not all findings are supportive of the autism spectrum hypothesis. Especially studies focusing on the link between executive functioning and autistic traits in TD individuals are inconsistent (Kunihira et al. 2006; Maes et al. 2012; Ridley et al. 2011).

In contrast to the inconsistent findings in linking the amount of autistic traits in TD individuals to their level of executive functioning, studies focusing on the social domain have generally been successful. One example concerns a study on loneliness and social relationships in the broader autism phenotype. Jobe and White (2007) showed that non-clinical students who score higher on the AQ report having shorter duration friendships, lower motivation for making new friends and keeping old friends, and experience more loneliness. Another social skill that has been examined in light of the autism spectrum hypothesis is the capacity to infer someone's disposition, using cues such as facial expression and gaze direction. Focusing on the former, Poljac et al. (2013) examined identification and interpretation of facial expressions. They showed that TD individuals with a high-AQ score were less accurate in recognising negative facial expressions, such as anger, disgust and sadness. The high-AQ group also showed decreased sensitivity, as they not only were less accurate but required higher emotional content to recognise the negative emotions as well. No effects were found for positive emotions, such as happiness. These findings were similar to patterns found in individuals diagnosed with ASD (Gross 2004).

The second cue, gaze direction, also proved to be a subdomain of social functioning on which TD individuals with more autistic traits showed results similar to diagnosed individuals. Hudson et al. (2012) examined how good people are at implicitly learning the intentions of others. In the learning phase, participants watched a video of two actors oriented with their face towards the participant. The gaze of the actors was manipulated either to move away from the participant or to approach him or her. Emotion was manipulated as well to make the actors look either happy or angry. By combining these two manipulations, either a positive or a negative disposition towards the participants was conveyed. After the learning phase, participants completed a gaze-cueing task. Individuals exhibiting few autistic traits had implicitly learned the disposition information, as reflected by fast responses on the task. High-AQ individuals on the other hand had not learned about the dispositions, and had therefore not implicitly 
acquired social information. A second gaze direction experiment focused more on the difference between low-AQ and high-AQ individuals in reciprocating direct gaze (Chen and Yoon 2011). Participants watched a video in which actors discussed a neutral topic with their gaze either directed towards the camera or averted. Individuals with low AQ scores modulated their gaze as a function of the gaze direction of the actors, indicated by spending more time looking at the direct gaze than the averted gaze. This higher tendency to reciprocate direct gaze was not found in individuals scoring high on the autistic spectrum, as they spent an equal amount of time looking at direct and averted gaze.

Focusing more on overt social behaviours, Kira and Aoyama (2006) examined the relationship between autistic traits and educational success in a group of physical therapy students. Groups were determined based on success or failure in a clinical training course that required students to demonstrate social communicational capacities. The difference in AQ score between the two groups was significant, indicating that individuals who failed the course exhibited more autistic traits. In addition, seven out of eight students who had failed the course, and 28 out of 36 students who had passed the course, could correctly be classified as respectively failing or passing based on the amount of autistic traits they exhibited. This meant that, overall, AQ score correctly predicted failing or passing the course in $80 \%$ of cases. Another study focusing on overt behaviour used a scenario-based task to examine expressions of pro-social behaviour (Jameel et al. 2014). When confronted with a character in need of help, TD individuals with more autistic traits generated less pro-social behaviours, such as co-operating and sharing. When asked to select a response from a set of three possible behaviours (low/medium/high pro-social), individuals with more autistic traits opted more often for the low pro-social and less often for the high pro-social response. A second scenario-based study on the use of social rules confirmed that high-AQ individuals exhibit less pro-social behaviour (Jameel et al. 2015). These findings were consistent with studies showing impaired prosocial behaviour in adults (Matson et al. 2012) and children and adolescents (Goodman et al. 2010; Russell et al. 2012) from clinical ASD groups.

Although the discussed studies do link autistic traits and social functioning in a TD population, critical evaluation of their methodology reveals that they might in fact not support a broad autism phenotype. First, the studies focus on very specific outcome variables. Social functioning is a broad concept that encompasses all skills that facilitate both verbal and nonverbal interaction and communication with other individuals. This includes outcome variables such as verbal reciprocity, eye contact, pro-social behaviour, initiating contact with others and recognising emotions in others. The full concept is therefore not covered by only taking one specific social skill into account, which is the case for the majority of the discussed studies.
While they intend to focus on the broad autism phenotype, they fail to cover the broad range of behaviours and capacities that together form social functioning, a problem that is aggravated by the already limited number of studies examining the social domain of the broad autism phenotype.

Second, several studies examining the broad autism phenotype do not in fact examine a true broad phenotype but instead look at extremes within the TD population. With respect to studies examining the broad phenotype for social functioning, one study used both a regression and an extreme group approach (Jobe and White 2007), and three out of the seven discussed studies exclusively selected participants from the extreme ends of the distribution (Jameel et al. 2014, 2015; Poljac et al. 2013). This was also the case for 4 of the 10 cognitive, visuospatial, executive and motor function studies mentioned (Grinter et al. 2009; Kunihira et al. 2006; Poljac et al. 2012; Stewart et al. 2009) and for 9 of the 12 studies discussed in a meta-analysis specifically focused on visuospatial ability (Cribb et al. 2016). Instead of using regression or a median split to include all participants, most of these studies examining the broad autism phenotype recruited several hundred participants and used extreme cut-offs or the upper and lower percentiles of these large databases to select TD individuals with respectively very high and very low AQ scores. While this increases the chance of finding significant differences between groups, it raises the question what these studies are actually examining. The failure to take the large middle section of the spectrum into account implies that the autism spectrum is not completely covered. Instead of investigating whether someone with a certain AQ score has less problems in social functioning than someone with a somewhat higher AQ score, it is examined whether someone with an extremely low AQ score differs from someone with an extremely high score, which is not what the autism spectrum hypothesis encompasses. In case of visuospatial ability, it has already been demonstrated that in the existing literature, the superior visuospatial skills found in individuals with ASD are only evident across the broad spectrum when comparing extreme groups of TD individuals (Cribb et al. 2016). This indicates that positive findings from studies using extremes do not necessarily replicate when examining a true broad spectrum consisting of a natural range of AQ scores. This could apply to the social domain as well. In addition, focusing on the upper extremes might bring bias into the sample. Individuals who score in the upper percentiles regularly have scores that are equal to those found in diagnosed individuals. If people have not been diagnosed despite of exhibiting many autistic traits, they may possess specific compensatory skills that help them function at a level on which a clinical ASD diagnosis is not suspected. This implies that a high-AQ group might not only quantitatively but also qualitatively differ from other individuals across the spectrum, thereby in fact disproving instead of supporting a broad autism phenotype. However, it must be acknowledged 
that reanalysis of the literature on visuospatial ability shows that removing individuals scoring above a clinical cut-off does not change the pattern of results, with high-AQ individuals and low-AQ individuals still significantly differing from each other (Cribb et al. 2016). It is, however, not clear whether this would also be the case for e.g. the social domain.

The present study accommodates for the discussed problems by employing a wider measure of social functioning (thereby solving the limited outcome problem) and by using AQ scores from all recruited TD participants (thereby solving the extreme score problem). Regression analyses were used to determine whether autistic traits across a TD population predict the frequency with which individuals exhibit a broad array of social behaviours and to predict the level of discomfort they experience when doing so. To provide a more finegrained overview of the relationship between social functioning and autistic traits among TD individuals, a separate correlation analysis on all AQ and social functioning sub-sores was performed as well. Based on the discussed studies, both regressions and most of the correlations were expected to yield substantial associations, thereby showing that AQ scores in a TD population are predictive of social functioning.

\section{Method}

\section{Participants}

Participants were 60 TD students from the Institute of Psychology who participated in reward for course credit. The sample consisted of 19 male and 41 female participants with a mean age of $M=20.25$ (SD =1.85), ranging from 18 to 26 years. Informed consent was obtained from all participants included in the study. All procedures performed were in accordance with the ethical standards of the institutional research committee and with the 1964 Helsinki Declaration and its later amendments.

\section{Procedure}

The used questionnaires were part of a larger study examining both behavioural and brain correlates of impairments often seen in ASD. The total experimental session took approximately $90 \mathrm{~min}$, including breaks. Completing the questionnaires was always done first.

\section{Measures}

Autism-Spectrum Quotient The Autism-Spectrum Quotient (AQ; Baron-Cohen et al. 2001) is a continuous and quantitative self-report measure of autistic traits in adults of normal intelligence. The questionnaire consists of 50 questions, divided into 5 sub-scales of 10 items each: social skill, attention switching, attention to detail, comunication, and imagination. Items are answered on a 4-point Likert-scale: definitely agree, slightly agree, slightly disagree, definitely disagree. Completing all items takes approximately $15 \mathrm{~min}$. Both the original English version of the test and its Dutch translation show satisfactory psychometric properties (Baron-Cohen et al. 2001; Hoekstra et al. 2008). The original scoring scheme as proposed by Baron-Cohen et al. (2001) is binary, which ignores the degree of agreement or disagreement. In line with Hoekstra et al. (2008), we included all four levels in scoring, which has been shown to improve the reliable range of measurement significantly (Murray et al. 2016). This resulted in a minimum total score of 50 (the individual reports having no autistic traits) and a maximum score of 200 (the individual reports having the full range of autistic traits). As could be expected based on score variability, reliability was better when using the full-range scoring scheme. Cronbach's alpha was $\alpha=0.82$ for the composite score (as opposed to $\alpha=0.71$ using binary scores), $\alpha=0.75$ ( $\alpha=0.55$ ) for social skill, $\alpha=0.73$ ( $\alpha=0.65$ ) for attention switching, $\alpha=.63(\alpha=0.53$ ) for attention to detail, $\alpha=0.62(\alpha=0.54)$ for communication, and $\alpha=0.46(\alpha=0.28)$ for imagination. These reliabilities were similar to those found by Hoekstra et al. (2008).

Inventory of Interpersonal Situations The Inventory of Interpersonal Situations (IIS; Van Dam-Baggen and Kraaimaat 1986) is a self-report questionnaire consisting of 35 interpersonal situations for which participants indicate on a 5-point scale how frequent they perform the described action (I never do - I always do) and how much discomfort they experience when doing so (no discomfort - very much discomfort). The items are split up into five sub-scales: criticising people (seven items), expressing your opinion (six items), giving compliments (four items), initiating contact (five items), and positive self-evaluation (four items). The remaining nine items form a residual category, with items touching upon topics such as saying no to requests from strangers and friends, asking people for help, asking people to return borrowed items, making eye-contact during conversation, asking for further explanation when not understanding something, and asking a friend to do a joint activity. Completing all items takes approximately $15 \mathrm{~min}$. The score range is $35-175$ for both the frequency and the discomfort scale. The psychometric characteristics of the questionnaire demonstrated to be good in both clinical and non-clinical samples (Van DamBaggen and Kraaimaat 1999). The data from the present study yielded a Cronbach's alpha of $\alpha=0.90$ for the frequency scale and $\alpha=0.95$ for the discomfort scale.

\section{Data Analyses}

Because both measures are the same type (self-report), there is a risk of Common Method Bias (CMB), in which variance in 
the data is attributable to the measurement method instead of the constructs that the measures are hypothesised to represent (Podsakoff and Organ 1986; Podsakoff et al. 2003, 2012). This can inflate or deflate correlations, thus leading to types I and II errors (Podsakoff et al. 2003, 2012; Williams and Brown 1994). Therefore, we first tested for CMB with the use of Harman's single-factor test.

Then, a set of preliminary analyses consisting of $t$ tests and ANOVAs examined whether the AQ and IIS scores differed between gender and median split based AQ groups, with tied scores assigned to the low-AQ group (resulting in unequal group sizes). The median split was primarily performed to compare the present findings with results from previous studies using extreme groups versus medium split based groups.

The broad autism phenotype for social functioning was tested with the use of two hierarchical multiple regression analyses (HMRAs) in which autistic traits predicted social functioning, controlling for age and gender. One regression predicted the reported frequency with which participants exhibited several social behaviours; a second regression predicted the level of discomfort participants reported to experience when doing so. A correlation analysis was performed to offer a more fine-grained overview of the relationship between all AQ and IIS sub-scores.

Across all analyses, an alpha level of $\alpha=0.05$ was used. In case of multiple comparisons, alpha was corrected using the Games-Howell procedure. If possible, effect sizes were reported as $\eta_{p}^{2}$ to facilitate comparison.

\section{Results}

\section{CMB}

All items (measuring latent variables) were loaded into an exploratory factor analysis. A fixed number of one factor was extracted. This was done separately for the relationship between the IIS frequency items and the AQ items on the one hand, and the IIS discomfort items and the AQ items on the other hand. The unrotated factor solutions were examined. For the analysis involving the IIS frequency items and the AQ items, $14.68 \%$ of variance was explained by the single factor. For the analysis involving the IIS discomfort items and the AQ items, this was $20.24 \%$. Since both values were below $50 \%$, we concluded that CMB was not an issue.

\section{Preliminary Analyses on the Autism-Spectrum Quotient}

The AQ scores ranged from 65 to 127 . The distribution was approximately normal as determined by visual inspection of the normal Q-Q plot. Table 1 shows the mean AQ scores and standard deviations per sex and group. Mean AQ score was comparable to findings from other social sciences students samples (Hoekstra et al. 2008). The low-AQ group and the high-AQ group did not significantly differ in age, $t(58)=1.16$, $p=.250, \eta_{p}^{2}=0.02$. The difference in AQ score between men and women was borderline significant, $t(58)=1.86, p=.068$, $\eta_{p}^{2}=0.06$, with men having higher scores than women. The median split-based groups significantly differed on AQ score, $t(58)=8.51, p<.001, \eta_{p}^{2}=0.56$, showing proper AQ score variation within the TD population.

To compare the present group means with the group means used in previous studies, a second composite AQ score and median split were calculated based on the binary scoring scheme used in most studies. The total AQ binary was $M=11.62$ ( $\mathrm{SD}=4.97)$. The low- $\mathrm{AQ}_{\text {binary }}$ group had a mean score of $M=8.09$ ( $\mathrm{SD}=2.49)$; the high-AQ binary $_{\text {group had a }}$ mean score of $M=15.93$ ( $\mathrm{SD}=3.66)$. The difference between the groups was 7.84, which was comparable to the difference reported in the study by Hudson et al. (2012), 7.60, who also used a median split. It was much smaller than the difference found in studies using extreme values (23.87 in Jameel et al. 2014; 24.37 in Jameel et al. 2015; 22.31 in Poljac et al. 2013).

\section{Preliminary Analyses on the Interpersonal Situations Frequency Score}

The IIS frequency scores ranged from 84 to 146 . The distribution was approximately normal as determined by visual inspection of the normal Q-Q plot. Table 2 shows the mean IIS frequency scores and standard deviations per sex and group. An independent samples $t$ test showed that the IIS frequency score of the low-AQ group was significantly higher than the IIS score of the high-AQ group, $t(58)=3.49, p=.001$, $\eta_{p}^{2}=0.17$. IIS score did not differ between men and women, $t(58)=1.02, p=.311, \eta_{p}^{2}=0.02$. Looking closely at the mean scores in Table 2, it is evident that especially women in the high-AQ group reported exhibiting few social behaviours. This was confirmed by a one-way ANOVA comparing the four groups, $F(3,56)=8.78, p<.001, \eta_{p}^{2}=0.32$. High-AQ women differed significantly from low-AQ women $(p<.001)$
Table 1 Mean (SD) AQ scores per gender and group

\begin{tabular}{llll}
\hline & Low-AQ & High-AQ & Total \\
\hline Men & $88.00(7.77) n=7$ & $106.58(8.51) n=12$ & $99.74(12.22) n=19$ \\
Women & $87.19(8.49) n=26$ & $104.60(8.09) n=15$ & $93.56(11.83) n=41$ \\
Total & $87.36(8.23) n=33$ & $105.48(8.18) n=27$ & $95.52(12.20) n=60$ \\
\hline
\end{tabular}


Table 2 Mean (SD) IIS frequency scores per gender and group

\begin{tabular}{llcl}
\hline & Low-AQ & High-AQ & Total \\
\hline Men & $117.00(16.88) n=7$ & $116.00(9.62) n=12$ & $116.37(12.32) n=19$ \\
Women & $119.65(13.84) n=26$ & $98.93(10.96) n=15$ & $112.07(16.24) n=41$ \\
Total & $119.09(14.29) n=33$ & $106.52(13.36) n=27$ & $113.43(15.14) n=60$ \\
\hline
\end{tabular}

and high-AQ men $(p=.001)$, but not from low-AQ men $(p=.115)$.

\section{Preliminary Analyses on the Interpersonal Situations Discomfort Score}

The IIS discomfort scores ranged from 44 to 136 . The distribution was positively skewed as determined by visual inspection of the normal Q-Q plot. Therefore, all analyses involving the IIS discomfort score were bootstrapped (1000 samples) to calculate corrected confidence intervals. Table 3 shows the mean IIS discomfort scores and standard deviations per sex and group. An independent samples $t$ test showed that the IIS discomfort score of the low-AQ group was significantly lower than the IIS score of the high-AQ group, $t(35.43)=3.16$, $p=.003,95 \%$ BCa CI $[6.84,26.02], \eta_{p}^{2}=0.16$. In addition, women reported to experience more discomfort than men, $t(53.93)=2.45, p=.017,95 \%$ BCa CI $[2.11,20.13]$, $\eta_{p}^{2}=0.07$. Looking closely at the mean scores in Table 3 , it is evident that especially women in the high-AQ group experienced much discomfort. This was confirmed by a one-way ANOVA comparing the four groups, $F(3,56)=9.94, p<.001$, $\eta_{p}^{2}=0.35$. High-AQ women differed significantly from lowAQ women ( $p=.010,95 \%$ BCa CI [5.67, 45.88]), low-AQ men $(p=.006,95 \%$ BCa CI $[8.12,55.67])$, and high-AQ men $(p=.019,95 \%$ BCa CI $[3.49,46.11])$.

\section{Do Autistic Traits Predict How Often People Exhibit Social Behaviours (IIS Frequency Score) After Controlling for Age and Gender?}

The HMRA showed that age and gender together did not significantly contribute to the regression model, $F(2$, $57)=0.57, p=.570, R^{2}=0.02$. A model with AQ score included as well did reach significance, $F(3,56)=9.46$, $p<.001, R^{2}=0.34$. Including AQ score in the model explained an additional $31.68 \%$ of variance, which was significant, $F_{\text {change }}(1,56)=26.74, p<.001$. The predictor coefficients of both models are shown in Table 4. AQ score and IIS frequency score were inversely related to each other: individuals with more autistic traits reported to exhibit less social behaviours, $t(56)=-5.17, p<.001$. This relationship is shown in Fig. 1 (solid line).

\section{Do Autistic Traits Predict How Much Discomfort People Experience When Engaging in Social Behaviours (IIS Discomfort Score) After Controlling for Age and Gender?}

The HMRA showed that age and gender together did not significantly contribute to the regression model, $F(2$, $57)=2.10, p=.132, R^{2}=0.07$. A model with AQ score included as well did reach significance, $F(3,56)=9.24$, $p<.001, R^{2}=0.33$. Including AQ score in the model explained an additional $26.27 \%$ of variance, which was significant, $F_{\text {change }}(1,56)=21.99, p<.001$. The predictor coefficients of both models are also shown in Table 4. Individuals with more autistic traits exhibited more discomfort when engaging in social behaviours, $t(56)=4.69,95 \%$ BCa CI $[0.55$, $1.24], p<.001$. This is also shown in Fig. 1 (dashed line). In addition, being female significantly predicted experiencing more discomfort in both model $1(p=.049)$ and model 2 $(p=.003)$.

\section{Correlating the AQ Scores with the IIS Frequency and Discomfort Scores}

Table 5 shows the correlations between the AQ scores and the frequency with which participants engaged in certain social behaviours and the level of discomfort they experienced when doing so. The AQ imagination scale and the AQ attention to detail scale were barely correlated with the frequency and discomfort scores: for the frequency sub-scales, only 4 out of the 14 correlations were significant; none of the correlations between AQ imagination and attention to detail on the one hand and the discomfort scores on the other hand reached significance. This meant that the frequency of engaging in social behaviours and the accompanying discomfort when doing so were not related to impaired imagination and to a detail-
Table 3 Mean (SD) IIS discomfort scores per gender and group

\begin{tabular}{llll}
\hline & Low-AQ & High-AQ & Total \\
\hline Men & $59.57(13.60) n=7$ & $66.67(12.48) n=12$ & $64.05(13.01) n=19$ \\
Women & $65.69(10.80) n=26$ & $91.47(26.11) n=15$ & $75.12(21.67) n=41$ \\
Total & $64.39(11.50) n=33$ & $80.44 .52(24.30) n=27$ & $71.62(19.92) n=60$ \\
\hline
\end{tabular}


Table 4 Hierarchical multiple regression models predicting IIS frequency and discomfort score

\begin{tabular}{lllrrrr}
\hline & & \multicolumn{1}{c}{$b$} & Beta & \multicolumn{1}{l}{$t$} \\
\hline Frequency & \multirow{2}{*}{ Model 1 } & Age & 0.36 & 0.04 & 0.32 & .747 \\
& & Gender & -3.90 & -0.12 & -0.88 & .380 \\
& \multirow{2}{*}{ Model 2 } & Age & 1.84 & 0.22 & 1.89 & .064 \\
& & Gender & -6.92 & -0.22 & -1.87 & .067 \\
Discomfort & \multirow{4}{*}{ Model 1 } & AQ score & -0.75 & -0.61 & -5.17 & $<.001$ \\
& & Age & 0.26 & 0.02 & 0.18 & .856 \\
& \multirow{4}{*}{ Model 2 } & Gender & 11.36 & 0.27 & 2.01 & .049 \\
& & Age & -1.51 & -0.14 & -1.17 & .245 \\
& & Gender & 14.98 & 0.35 & 3.06 & .003 \\
& & AQ score & 0.90 & 0.55 & 4.69 & $<.001$
\end{tabular}

Frequency: $R^{2}=0.02$ for model $1, \Delta R^{2}=0.32$ for model $2(p<.001)$; discomfort: $R^{2}=0.07$ for model $1, \Delta R^{2}=0.26$ for model $2(p<.001)$

focused processing style. Both the frequency scores and the discomfort scores were primarily related to the AQ scores for social skill and attention switching, indicating that a higher level of autistic traits in those domains was related to engaging in less social behaviours and experiencing more discomfort. Most of the correlations between frequency and discomfort scores on the one hand and AQ social skills and attention switching on the other hand were medium $(r=.30)$ to large $(r=.50)$. The frequency of criticising others was not significantly related to any of the AQ scores, having only small $(r=.10)$ to medium $(r=.30)$ correlations. However, individuals scoring high on the AQ social skills and attention switching sub-scales did experience more discomfort when criticising others $(r=.29$ for social skills and $r=.31$ for attention switching, respectively). A final notable pattern was that of the AQ communication sub-scale, which correlated significantly with the same frequency and discomfort subscales: giving compliments, initiating contact and positive self-evaluation.

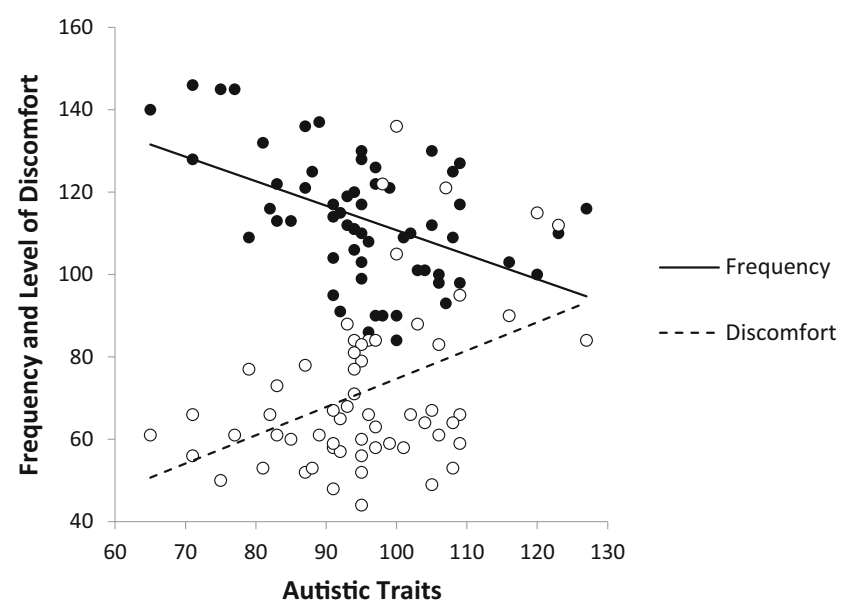

Fig. 1 The relationship between autistic traits ( $x$-axis) and the frequency and discomfort of social behaviours ( $y$-axis)

\section{Discussion}

The present study extended the research on the broad autism phenotype for social functioning by using a measure that incorporated several sub-domains of social functioning and by including TD individuals who exhibited a natural range of autistic traits. This accommodated for two problems observed in previous studies: only focusing on one specific indicator of social functioning and only including TD individuals with extreme AQ scores.

The present findings show that social functioning varies across the autistic spectrum in a non-clinical population, thereby supporting the notion that individual differences in social functioning are related to the broad autism phenotype. Regression analyses showed that autistic traits within the TD population predict both the frequency with which individuals engage in several different social behaviours and the level of discomfort they experience when doing so, both after controlling for age and gender. Having more autistic traits predicted exhibiting less social behaviours and experiencing more discomfort. In addition, correlation analyses showed that almost all queried social behaviours were related to one or more specific autistic traits. Especially the social skills and attention switching AQ sub-scores were strongly related to many social behaviours and discomfort indicators.

The current findings on the broad autism phenotype are consistent with previous studies linking the amount of autistic traits individuals exhibit to several sub-domains of social functioning, such as initiating and maintaining relationships (Jobe and White 2007), recognising facial expressions (Poljac et al. 2013), using gaze direction information (Hudson et al. 2012), reciprocating direct gaze (Chen and Yoon 2011), displaying social communicational capacities (Kira and Aoyama 2006), and exhibiting pro-social behaviour (Jameel et al. 2014, 2015). In addition, the current findings provide a stronger test of the broad phenotype than most of these previous studies by covering more social behaviours and by including a more natural range of AQ scores. The fact that the relationship between autistic traits and social functioning remained intact after resolving the limited outcome problem and the extreme score problem supports the existence of a broad autism phenotype.

The finding that both the frequency and the discomfort score were related strongest to the AQ social skill sub-score is not surprising, since this scale shows the largest conceptual overlap with the frequency and discomfort measures. This explanation however implies the risk that the significant result of the regression analyses was caused by only one sub-scale of the predictor overlapping the outcome variable very closely. To rule out this possibility, both the frequency and the discomfort regression analyses were repeated two times. First, the predictor variable was changed into a composite AQ score but without the AQ social skill sub-score included. Though 
Table 5 Correlations $(N=60)$ between AQ sub-scores and IIS scores for six social functioning scales

\begin{tabular}{|c|c|c|c|c|c|c|}
\hline & $\begin{array}{l}\text { AQ } \\
\text { social } \\
\text { skills }\end{array}$ & $\begin{array}{l}\text { AQ attention } \\
\text { switching }\end{array}$ & $\begin{array}{l}\text { AQ } \\
\text { communication }\end{array}$ & $\begin{array}{l}\mathrm{AQ} \\
\text { imagination }\end{array}$ & $\begin{array}{l}\text { AQ attention } \\
\text { to detail }\end{array}$ & $\begin{array}{l}\mathrm{AQ} \\
\text { total }\end{array}$ \\
\hline \multicolumn{7}{|l|}{ IIS frequency } \\
\hline $\begin{array}{l}\text { Criticising } \\
\text { people }\end{array}$ & -0.14 & -0.23 & -0.12 & -0.20 & 0.04 & -0.20 \\
\hline $\begin{array}{c}\text { Expressing } \\
\text { opinions }\end{array}$ & $-0.38 * *$ & -0.24 & -0.10 & -0.12 & -0.04 & $-0.28^{*}$ \\
\hline $\begin{array}{l}\text { Giving } \\
\text { compli- } \\
\text { ments }\end{array}$ & $-0.26^{*}$ & $-0.26^{*}$ & $-0.35 * *$ & -0.23 & 0.07 & $-0.31^{*}$ \\
\hline $\begin{array}{r}\text { Initiating } \\
\text { contact }\end{array}$ & $-0.48^{*}$ & $-0.35^{* *}$ & $-0.29^{*}$ & -0.02 & -0.05 & $-0.38 * *$ \\
\hline $\begin{array}{l}\text { Positive } \\
\text { self-- } \\
\text { evaluation }\end{array}$ & $-0.41^{* *}$ & $-0.37 * *$ & $-0.40 * *$ & $-0.40 * *$ & -0.07 & $-0.50 * *$ \\
\hline $\begin{array}{l}\text { Residual } \\
\text { items }\end{array}$ & $-0.37^{* *}$ & $-0.30^{*}$ & -0.21 & $-0.37 * *$ & $-0.29^{*}$ & $-0.47 * *$ \\
\hline $\begin{array}{l}\text { Frequency } \\
\text { total }\end{array}$ & $-0.46^{* *}$ & $-0.39 * *$ & $-0.31^{*}$ & $-0.30 *$ & -0.10 & $-0.48 * *$ \\
\hline \multicolumn{7}{|l|}{ IIS discomfort } \\
\hline $\begin{array}{l}\text { Criticising } \\
\text { people }\end{array}$ & $0.29 *$ & $0.31 *$ & 0.11 & 0.05 & 0.02 & 0.25 \\
\hline $\begin{array}{c}\text { Expressing } \\
\text { opinions }\end{array}$ & $0.29 *$ & $0.27 *$ & 0.04 & -0.03 & $<0.01$ & 0.19 \\
\hline $\begin{array}{l}\text { Giving } \\
\text { compli- } \\
\text { ments }\end{array}$ & $0.34 * *$ & $0.31^{*}$ & $0.34 * *$ & 0.23 & 0.10 & $0.40 * *$ \\
\hline $\begin{array}{r}\text { Initiating } \\
\text { contact }\end{array}$ & $0.62 * *$ & $0.37 * *$ & $0.31 *$ & -0.02 & -0.01 & $0.41 * *$ \\
\hline $\begin{array}{l}\text { Positive } \\
\text { self-- } \\
\text { evaluation }\end{array}$ & $0.40 * *$ & $0.36 * *$ & $0.36^{*}$ & 0.14 & 0.12 & $0.43 * *$ \\
\hline $\begin{array}{l}\text { Residual } \\
\text { items }\end{array}$ & $0.43^{*}$ & $0.44 * *$ & 0.23 & 0.23 & 0.13 & $0.46^{* *}$ \\
\hline $\begin{array}{l}\text { Discomfort } \\
\text { total }\end{array}$ & $0.47 * *$ & $0.42 * *$ & 0.25 & 0.11 & 0.07 & $0.42 * *$ \\
\hline
\end{tabular}

* significant at .05 level (two-tailed); ** significant at .01 level (two-tailed) the exact test statistics changed, none of the conclusions from the original analyses as reported in the results section (see Table 4) changed. Model 1 was still not significant, and adding the AQ score still significantly improved the prediction into a significant model 2 . Thus, by not including the variance caused by the AQ social skill sub-score in the predictor, autistic traits still significantly predicted social functioning. A second test of the influence of the AQ social skill sub-score was done by including the sub-score in the analysis and controlling for it in the regression. The influence of the social skill subscore was clearly seen in that the first model was now significant. However, adding the composite AQ score still significantly improved the prediction.

Just as the social skill sub-scale, the attention switching sub-scale strongly correlated with both the IIS frequency and the discomfort score. Since attention switching can be considered a cognitive rather than a social construct, this finding seems not straightforward. However, there are several reasons why it is more straightforward than it would seem. First, there is partial conceptual overlap; attention switching does include attention shifts not only between non-social stimuli but also between social stimuli. A clear example is formed by item 10 of the AQ: 'in a social group, I can easily keep track of several different people's conversations'. Related to this, the preference for sameness and the resistance to change characteristic of individuals high on the spectrum are also true for the social domain. Social interactions can be very changeable, which may explain the strong and consistent correlations between the AQ attention switching sub-scale and the IIS scores: dealing with social interaction generally requires the ability to quickly shift attention, and having a higher amount of autistic traits may therefore be related to displaying less social behaviours and experiencing more discomfort when doing so.

The communication sub-scale showed a very specific correlation pattern, correlating significantly with giving compliments and initiating contact and positive self-evaluation for 
both frequency and discomfort. Compared to the nonsignificant correlations (criticising people and expressing opinions), the significant sub-scales seem much more characteristic of the social impairments individuals with ASD generally face, namely social skills focused on 'reading' how others might respond and act in social situations. This supports the validity of the AQ in measuring social skill impairments seen in ASD.

Two AQ sub-scales barely correlated with the IIS frequency and discomfort scores: the imagination and attention to detail sub-scales. The low correlations with the attention to detail sub-scale can be explained relatively easily, since this sub-scale mostly focuses on cognitive rather than social characteristics, and shows little conceptual overlap with the social domain. The low correlations with the imagination sub-scale might arise from the low reliability this sub-scale showed. An alternative explanation touches more upon the core of the imagination concept. While imagination covers combining ideas and information to form a mental image of every entity you can think of, Ten Eycke and Müller (2015) state that the general idea of impaired imagination in ASD comes from measurement instruments focusing exclusively on social imagination. This would mean that the reported imagination impairment could also be explained by the social difficulties individuals with ASD exhibit. Ten Eycke and Müller (2015) provided early evidence for this hypothesis by examining both person- and object-focused imagination in children, indeed finding support for person-focused imagination impairments but not for object-focused ones. This is consistent with clinical observations of the first author. The findings imply that if the present measure of imagination is less dependent on social imagination, the correlation between social functioning and the imagination sub-score may indeed be low. Close examination of the items of the AQ imagination sub-scale showed that approximately half of the items focuses on social imagination. The other half is more focused on objects or general scenes, for which imagination is not impaired, according to Ten Eycke and Müller (2015). This could explain why the imagination score was not significantly correlated with most frequency and discomfort scores.

One notable finding was not covered by the core hypotheses of the current study: the social functioning of women with higher AQ scores. Women who scored high on the AQ reported exhibiting significantly less social behaviours and experiencing more discomfort. Close examination of these results showed that women who score higher on the autistic spectrum not only are more impaired in social functioning than individuals with less autistic traits but also compared to men with an equal amount of autistic traits. Research on ASD in women indicates that compared to men with ASD, women are less withdrawn and display more TD-like social behaviour (Spek 2013). This is often explained by an interaction between societal pressure and compensatory mechanisms. Females are expected to be more social, nurturing and empathetic. This causes girls with ASD to already feel different at a young age, and makes them develop social tricks, such as standard conversation starters and mimicking gaze patterns, in order to live up to those standards (Spek 2013; Spek and Goosen 2010). Employing these compensatory mechanisms results in relatively successful masking of social-communicative impairments, but demands a lot of (mental) effort, which means that they often require time to recover from social interactions (Spek and Goosen 2013). This latter finding could explain the higher level of discomfort women with higher AQ scores experience: their attempts to mask their social impairments cost effort, which is reflected in more discomfort. In contrast, the present observation of fewer social behaviours in high-scoring women seems to be the opposite of what previous research would predict (Spek 2013). This discrepancy could be explained by the aforementioned societal expectations on social behaviour in women. If women are expected to be more sociable than men, it is possible that women judge themselves against a different standard than men, which leads to lower self-reported estimates of exhibited social behaviours. Men and women then do not differ in the amount of social behaviours they exhibit, but report on this differently because of a societal-induced gender-specific self-report bias. One possibility to avoid this issue could be the use of observation instead of self-report. However, since high-AQ women employ compensatory mechanisms to cover up at least part of their social-communicational deficits, the use of observation would not give an accurate estimate either. Another possibility is presenting participants with a more a clear-cut reporting strategy, such as daily reporting on the exact number of times every social behaviour on the list was performed. Though examining TD women with high-AQ scores was not the central aim of the present study, the finding is notable and should be examined more closely in future studies.

Returning to the general aim of the present study, certain limitations should be discussed. First, the research is completely based on self-report measures. While this does not have to be a problem, it could bring bias into the data, such as the discussed possible societal-induced gender-specific self-report bias with regard to the findings on high-AQ women. However, we also discussed that other measures, such as observation, do not always provide a sufficient fix, since these measures are characterised by their own limitations. Related to this first limitation, we must acknowledge that the AQ in specific might be sub-optimal relative to other selfreport measurements of autism traits. Ingersoll et al. (2011) e.g. showed that the $A Q$, compared to two other self-report measures, scores worse on internal consistency, proposed factor structure, and criterion validity. However, performing worse did not mean performing bad. In addition, the AQ is the most-used self-report measure for autistic traits in a TD population, making easy comparison between the present 
findings and the previous studies on the broad autism phenotype a clear advantage of employing this measure. A third limitation concerns the sample size, which could be argued to be relatively low. Whereas larger samples are indeed generally better, a post-hoc power analysis on the two main regression analyses (assuming an $\alpha$ error probability of 0.05 ) showed that both analyses had sufficient power $(1-\beta=0.97)$. So, the used sample size was certainly sufficient to answer the main research questions. Fourth, we did not examine the whole spectrum, since no clinical individuals were included. Future research should therefore include both a clinical and a non-clinical sample. Last, we must acknowledge that although the present study examined a large part of the social domain, taking several social skills into account and examining both frequency and discomfort, we did not address all different aspects of social functioning. The solution to this is related to the already discussed research paucity on how social skills relate to the broad autism phenotype.

We showed that the broad autism phenotype concept is still supported when taking into account the two limitations of previous research: only covering a limited range of social behaviours and only including participants with extreme AQ scores. Using this knowledge will be more pertinent for the latter than the former limitation. The current study employed a questionnaire so that a broad range of social behaviours could be examined. We certainly acknowledge the importance of looking at specific social skills, and examining a broad range of social behaviours was done mostly because of the limited number of studies on specific social skills available within the literature. As more studies emerge on the relationship between autistic traits and social functioning across a broad spectrum, it will be less necessary to use overarching measures, and it will become more informative to focus on specific social behaviours. However, judging by the research paucity in this area, this may take time.

With regard to the latter limitation, we hope that future studies will continue to explore the broad autism spectrum using an actual range of autistic traits instead of two extremes. However, we acknowledge that the choice of either comparing extreme groups or using a continuous measure in general falls within a broader discussion, in which both options are associated with pros and cons. In their comprehensive evaluation of the benefits and costs associated with using the extreme groups approach, Preacher et al. (2005) mention several reasons for using extreme groups, most importantly costefficiency and increased power. So, the use of extreme groups could be considered in cases where it is not feasible to recruit a full range continuous sample or in areas where the hypothesised effect is small. One should however be careful not to fall victim to post-hoc sub-grouping: obtaining data on both the dependent and independent variable across the full spectrum, but analysing only data from individuals scoring at the extremes (Preacher et al. 2005). This practice does not improve cost-efficiency, and does not lead to the power improvement associated with the extreme groups approach. Since post-hoc sub-grouping was not the case for the extreme group studies in the social domain discussed in the present article (Jameel et al. 2014, 2015; Poljac et al. 2013), we can conclude that the extreme groups approach was applied appropriately. However, interpretation of the results of these studies should still take the disadvantages of using extreme groups into account, such as problems associated with regression to the mean (Campbell and Kenny 1999), increased chances of model misspecification because of unwarranted assumptions about linearity (Feldt 1961), reduction of individual differences information, and possibly reduced reliability (Preacher et al. 2005). So, if a continuous approach is feasible within a given research context, we strongly advise applying it. This is true not only for the social domain but for the cognitive and motor domain. If findings in other domains of functioning in ASD are equally supportive of the broad phenotype as the present findings, applying a continuous approach would provide strong proof for the notion of a spectrum covering every single individual. This challenges the traditional, categorical view in favour of a more quantitative approach, and changes the way we approach the concept of normality.

Acknowledgements We thank two anonymous reviewers for their helpful comments on earlier drafts of this manuscript.

Authors' Contributions KdG conceived of the study, participated in its design, performed the measurements and statistical analyses and interpreted the data. JvS participated in the design and coordination of the study, participated in analysing the data and revised the manuscript critically for important intellectual content. All authors read and approved the final manuscript.

Compliance with Ethical Standards Informed consent was obtained from all participants included in the study. All procedures performed were in accordance with the ethical standards of the institutional research committee and with the 1964 Helsinki Declaration and its later amendments.

Open Access This article is distributed under the terms of the Creative Commons Attribution 4.0 International License (http:// creativecommons.org/licenses/by/4.0/), which permits unrestricted use, distribution, and reproduction in any medium, provided you give appropriate credit to the original author(s) and the source, provide a link to the Creative Commons license, and indicate if changes were made.

\section{References}

American Psychiatric Association. (2000). Diagnostic and statistical manual of mental disorders (4th ed., text rev.). Washington, DC: Author.

American Psychiatric Association. (2013). Diagnostic and statistical manual of mental disorders (5th ed.). Washington, DC: Author. 
Bailey, A., Palferman, S., Heavey, L., \& Le Couteur, A. (1998). Autism: the phenotype in relatives. Journal of Autism and Developmental Disorders, 28, 369-392.

Baron-Cohen, S., Bolton, P., Wheelwright, S., Scahill, V., Short, L., Mead, G., \& Smith, A. (1998). Autism occurs more often in families of physicists, engineers, and mathematicians. Autism, 2, 296-301.

Baron-Cohen, S., Wheelwright, S., Scott, C., Bolton, P., \& Goodyer, I. (1997). Is there a link between engineering and autism? Autism, 1, 153-163.

Baron-Cohen, S., Wheelwright, S., Skinner, R., Martin, J., \& Clubley, E. (2001). The autism-spectrum quotient (AQ): evidence from Asperger syndrome/high-functioning autism, males and females, scientists and mathematicians. Journal of Autism and Developmental Disorders, 31, 5-17.

Bayliss, A. P., \& Kritikos, A. (2011). Brief report: perceptual load and the autism spectrum in typically developed individuals. Journal of Autism and Developmental Disorders, 41, 1573-1578.

Bishop, D. V., Maybery, M., Maley, A., Wong, D., Hill, W., \& Hallmayer, J. (2004). Using self-report to identify the broad phenotype in parents of children with autistic spectrum disorders: a study using the autism-spectrum quotient. Journal of Child Psychology and Psychiatry, 45, 1431-1436.

Campbell, D. T., \& Kenny, D. A. (1999). A primer on regression artifacts. New York: Guilford Press.

Chen, F. S., \& Yoon, J. M. (2011). Brief report: broader autism phenotype predicts spontaneous reciprocity of direct gaze. Journal of Autism and Developmental Disorders, 41, 1131-1134.

Constantino, J. N., \& Todd, R. D. (2003). Autistic traits in the general population: a twin study. Archives of General Psychiatry, 60, 524530 .

Cribb, S. J., Olaithe, M., Di Lorenzo, R., Dunlop, P. D., \& Maybery, M. T. (2016). Embedded figures test performance in the broader autism phenotype: a meta-analysis. Journal of Autism and Developmental Disorders, 46, 2924-2939.

DiCriscio, A. S., \& Troiani, V. (2017). Brief report: autism-like traits are associated with enhanced ability to disembed visual forms. Journal of Autism and Developmental Disorders, 47, 1-9.

Feldt, L. S. (1961). The use of extreme groups to test for the presence of a relationship. Psychometrika, 26, 307-316.

Fugard, A. J., Stewart, M. E., \& Stenning, K. (2011). Visual/verbalanalytic reasoning bias as a function of self-reported autistic-like traits: a study of typically developing individuals solving Raven's advanced progressive matrices. Autism, 15, 327-340.

Goodman, A., Lamping, D. L., \& Ploubidis, G. B. (2010). When to use broader internalising and externalising subscales instead of the hypothesised five subscales on the strengths and difficulties questionnaire (SDQ): data from British parents, teachers and children. Journal of Abnormal Child Psychology, 38, 1179-1191.

Grinter, E. J., Van Beek, P. L., Maybery, M. T., \& Badcock, D. R. (2009). Brief report: visuospatial analysis and self-rated autistic-like traits. Journal of Autism and Developmental Disorders, 39, 670-677.

Gross, T. F. (2004). The perception of four basic emotions in human and nonhuman faces by children with autism and other developmental disabilities. Journal of Abnormal Child Psychology, 32, 469-480.

Hoekstra, R. A., Bartels, M., Cath, D. C., \& Boomsma, D. I. (2008). Factor structure, reliability and criterion validity of the autismspectrum quotient (AQ): a study in Dutch population and patient groups. Journal of Autism and Developmental Disorders, 38, $1555-1566$

Hudson, M., Nijboer, T. C., \& Jellema, T. (2012). Implicit social learning in relation to autistic-like traits. Journal of Autism and Developmental Disorders, 42, 2534-2545.

Ingersoll, B., Hopwood, C. J., Wainer, A., \& Donnellan, M. B. (2011). A comparison of three self-report measures of the broader autism phenotype in a non-clinical sample. Journal of Autism and Developmental Disorders, 41, 1646-1657.
Jameel, L., Vyas, K., Bellesi, G., Cassell, D., \& Channon, S. (2015). Great expectations: The role of rules in guiding pro-social behaviour in groups with high versus low autistic traits. Journal of Autism and Developmental Disorders, 45, 2311-2322.

Jameel, L., Vyas, K., Bellesi, G., Roberts, V., \& Channon, S. (2014). Going 'above and beyond': are those high in autistic traits less pro-social? Journal of Autism and Developmental Disorders, 44, $1846-1858$.

Jobe, L. E., \& White, S. W. (2007). Loneliness, social relationships, and a broader autism phenotype in college students. Personality and Individual Differences, 42, 1479-1489.

Kira, Y., \& Aoyama, H. (2006). The autism-spectrum quotient score of students who failed clinical training. Aino Journal, 5, 51-56.

Kraepelin, E. (1917). Lectures on clinical psychiatry (3rd ed.). New York, NY: William Wood.

Kunihira, Y., Senju, A., Dairoku, H., Wakabayashi, A., \& Hasegawa, T. (2006). 'Autistic' traits in non-autistic Japanese populations: relationships with personality traits and cognitive ability. Journal of Autism and Developmental Disorders, 36, 553-566.

Maes, J. H., Vissers, C. T., Egger, J. I., \& Eling, P. A. (2012). On the relationship between autistic traits and executive functioning in a non-clinical Dutch student population. Autism, 17, 379-389.

Matson, J. L., Hattier, M. A., \& Turygin, N. (2012). An evaluation of social skills in adults with pica, autism spectrum disorders, and intellectual disability. Journal of Developmental and Physical Disabilities, 24, 505-514.

Murray, A. L., Booth, T., McKenzie, K., \& Kuenssberg, R. (2016). What range of trait levels can the autism-spectrum quotient (AQ) measure reliably? An item response theory analysis. Psychological Assessment, 28, 673-683.

Piven, J. (2001). The broad autism phenotype: a complementary strategy for molecular genetic studies of autism. American Journal of Medical Genetics, 105, 34-35.

Podsakoff, P. M., MacKenzie, S. B., Lee, J., \& Podsakoff, N. P. (2003). Common method biases in behavioral research: a critical review of the literature and recommended remedies. Journal of Applied Psychology, 88, 879-903.

Podsakoff, P. M., MacKenzie, S. B., \& Podsakoff, N. P. (2012). Sources of method bias in social science research and recommendations on how to control it. Annual Review of Psychology, 63, 539-569.

Podsakoff, P. M., \& Organ, D. W. (1986). Self-reports in organizational research: problems and prospects. Journal of Management, 12, 531544.

Poljac, E., Poljac, E., \& Yeung, N. (2012). Cognitive control of intentions for voluntary actions in individuals with a high level of autistic traits. Journal of Autism and Developmental Disorders, 42, 2523-2533.

Poljac, E., Poljac, E., \& Wagemans, J. (2013). Reduced accuracy and sensitivity in the perception of emotional facial expressions in individuals with high autism spectrum traits. Autism, 17, 668-680.

Preacher, K. J., Rucker, D. D., MacCallum, R. C., \& Nicewander, W. A. (2005). Use of the extreme groups approach: a critical reexamination and new recommendations. Psychological Methods, 10, 178-192.

Remington, A., Swettenham, J., Campbell, R., \& Coleman, M. (2009). Selective attention and perceptual load in autism spectrum disorder. Psychological Science, 20, 1388-1393.

Ridley, N. J., Homewood, J., \& Walters, J. (2011). Cerebellar dysfunction, cognitive flexibility and autistic traits in a non-clinical sample. Autism, 15, 728-745.

Russell, G., Golding, J., Norwich, B., Emond, A., Ford, T., \& Steer, C. (2012). Social and behavioural outcomes in children diagnosed with autism spectrum disorders: A longitudinal cohort study. Journal of Child Psychology and Psychiatry, 53, 735-744.

Ruta, L., Mazzone, D., Mazzone, L., Wheelwright, S., \& Baron-Cohen, S. (2012). The autism-spectrum quotient-Italian version: a cross- 
cultural confirmation of the broader autism phenotype. Journal of Autism and Developmental Disorders, 42, 625-633.

Ruzich, E., Allison, C., Smith, P., Ring, H., Auyeung, B., \& BaronCohen, S. (2016). The autism-spectrum quotient in siblings of people with autism. Autism Research, 1, 1-9.

Scheeren, A. M., \& Stauder, J. E. (2008). Broader autism phenotype in parents of autistic children: reality or myth? Journal of Autism and Developmental Disorders, 38, 276-287.

Shah, A., \& Frith, U. (1983). An islet of ability in autistic children: a research note. Journal of Child Psychology and Psychiatry, 24, 613-620.

Shah, A., \& Frith, U. (1993). Why do autistic individuals show superior performance on the block design task? Journal of Child Psychology and Psychiatry, 34, 1351-1364.

Spek, A. A. (2013). Autismespectrumstoornissen bij meisjes en vrouwen [Autism spectrum disorders in girls and women]. Tijdschrift Voor Geneeskunde, 157, 1-6.

Spek, A. A., \& Goosen, A. C. A. (2010). Autismespectrumstoornissen bij vrouwen [Autism spectrum disorders in females]. Engagement, 1, $36-38$.

Spek, A. A., \& Goosen, A. C. A. (2013). Autismespectrumstoornissen bij meisjes en vrouwen, een eerste verkenning [Autism spectrum disorders in girls and females, an early examination]. Wetenschappelijk Tijdschrift Autisme, 2, 62-67.

Stewart, M. E., Watson, J., Allcock, A. J., \& Yaqoob, T. (2009). Autistic traits predict performance on the block design. Autism, 13, 133-142.
Szatmari, P., Paterson, A. D., Zwaigenbaum, L., Roberts, W., Brian, J., Liu, X., et al. (2007). Mapping autism risk loci using genetic linkage and chromosomal rearrangements. Nature Genetics, 39, 319-328.

Ten Eycke, K. D., \& Müller, U. (2015). Brief report: new evidence for a social-specific imagination deficit in children with autism spectrum disorder. Journal of Autism and Developmental Disorders, $45,213-220$.

Van Dam-Baggen, C. M. J., \& Kraaimaat, F. W. (1986). De betrouwbaarheid en validiteit van de inventarisatielijst omgaan met anderen [The reliability and validity of a self-report inventory for social anxiety]. Gedragstherapie, 19, 229-248.

Van Dam-Baggen, C. M. J., \& Kraaimaat, F. W. (1999). Assessing social anxiety: the inventory of interpersonal situations (IIS). European Journal of Psychological Assessment, 15, 25-38.

Wheelwright, S., Auyeung, B., Allison, C., \& Baron-Cohen, S. (2010). Defining the broader, medium and narrow autism phenotype among parents using the autism spectrum quotient (AQ). Molecular Autism, 1,10 .

Williams, L. J., \& Brown, B. K. (1994). Method variance in organizational behavior and human resources research: effects on correlations, path coefficients, and hypothesis testing. Organizational Behavior and Human Decision Processes, 57, 185-209.

Wing, L. (1988). The autistic continuum. In L. Wing (Ed.), Aspects of autism: biological research. London: Gaskell/Royal College of Psychiatrists. 